PFC/JA-94-6

\title{
Marfe Formation in Diverted Tokamaks
}

\author{
J. Kesner, J. P. Freidberg
}

MIT Plasma Fusion Center

Cambridge, Massachusetts 02139 USA

September 1994

This work was supported by the US Department of Energy under contract DE-FG02-91ER-54109. Reproduction, translation, publication, use, and disposal, in whole or in part, by or for the US Government is permitted.

Submitted for publication in: Nuclear Fusion 


\title{
DRAFT
}

\author{
Marfe Formation in Diverted Tokamaks \\ J. Kesner, J.P. Freidberg \\ Massachusetts Institute of Technology
}

September 21, 1994

Plasma Fusion Center report PFC/JA-94-6

\begin{abstract}
We consider the equilibrium and stability of Marfes. We demonstrate that important aspects of the 2-dimensional problem can be illustrated by a 1-dimensional treatment. A stability analysis indicates that Marfe formation requires low temperature, high impurity content and low cross field transport. Additionally, Marfes tend to form on flux surfaces that contain an x-point. We observe that the transition to a Marfe-like solution can result from a fluctuation-induced jump from a neighboring constant temperature solution.
\end{abstract}




\section{Introduction}

Marfes are radiation condensation instabilities that appear in tokamaks as a density limit is approached [1-5]. They are manifested as dense, cool regions of radiating plasma that form a poloidally localized and toroidally symmetric ring usually located near an outer flux surface on the high field side of the torus. Recent observations indicate that Marfes tend to locate themselves near to the $\mathrm{x}$-point [6-7] in a diverted tokamak as the edge is cooled and densified.

A number of numerical and analytic investigations of this phenomenon have been performed [8-12]. The tokamak thermal equilibrium equations are two dimensional, i.e. in the perpendicular and along-the-field-line co-ordinates (the perpendicular coordinate is dominantly the flux co-ordinate). McCarthy and Drake [10] replaced the cross-field heat flux by a constant heat source and solved the time dependent non-linear 1-dimensional along-the-field-line equation. They demonstrated a transition from an unstable isothermal solution into a Marfe-like solution. The two dimensional equations have also been studied [13-14].

In this work we make a more correct (albeit still simplistic) approximation to the cross field heat flux. We observe that the important behavior is along-the-field-line and that we can simplify the problem mathematically by differencing the cross-field diffusion term. This reduces the 2-dimensional partial differential equation to a set of coupled non-linear 1-dimensional ordinary differential equations. We can then solve the resulting non-linear along-the-field-line equations for equilibrium. We consider first the properties of a single ordinary differential equation which models the along-the-field-line heat flow in the vicinity of the $\mathrm{x}$-point. We then consider a set of two coupled ordinary differential equations which model respectively the heat flow in the separatrix and scrape-off layer flux surfaces.

Examination of a single along-the-field-line equation reveals several stable solutions which may be divided into two classes: 1) those that exhibit a constant temperature along the field line and 2) those in which temperature varies along the field line, i.e. exhibit a Marfe-like character. The constant temperature equilibria will make a transition into a 
Marfe if they become linearly unstable. Additionally, temperature fluctuations can cause a stable constant temperature equilibrium to "jump" into the Marfe-like solution when these two equilibria approach each other.

We find that this model for the transition can explain a number of observed phenomena. Formation of a stable Marfe-like solution can occur when the temperature and the cross field transport are sufficiently low and the impurity content is sufficiently high. The model is consistent with the tendency of an x-point to facilitate Marfe formation.

\section{Basic Equations}

The theory of Marfes is based on the fluid equations [15] for density ( $n$ ), temperature (T) and velocity $v_{\|}$. Assuming $T_{i}=T_{e}=T$ we will examine the equilibria of the following equations:

$$
\begin{gathered}
\frac{\partial}{\partial s} \kappa_{\|} \frac{\partial T}{\partial s}+\frac{\partial}{\partial x} \kappa_{\perp} \frac{\partial T}{\partial x}=n_{e} n_{C} L(T) . \\
n_{e} T=p(x)
\end{gathered}
$$

with $s$ and $x$ the respective along-the-field and cross-field directions, $\kappa_{\|}$and $\kappa_{\perp}$ the parallel and perpendicular thermal conductivities respectively, and $\mathrm{L}(\mathrm{T})$ the radiation function. We imagine that the field line length from outside to inside of the torus is $\mathrm{L}\left(\sim q_{a} R\right.$ with $q_{a}$ the safety factor near the plasma edge) and impose the symmetry boundary condition

$$
\frac{\partial T(x, 0)}{\partial s}=\frac{\partial T(x, L)}{\partial s}=0
$$

If we consider cylindrical co-ordinates we can use $\mathrm{r}$ as the radial (flux) coordinate, $\theta$ as the poloidal co-ordinate and $\phi$ as the toroidal co-ordinate. In this approximation $B_{\theta} / B_{\phi}=\tan \alpha$ with $\alpha$ the field line pitch angle and $L \approx \pi a \kappa / \sin \alpha$ with $\kappa$ the flux tube ellipticity and $\pi a \kappa$ approximately half of the flux tube circumference at the Marfe location. In the poloidal plane Eq. (1) becomes 


$$
\frac{1}{r^{2}} \frac{\partial}{\partial \theta}\left(\kappa_{\|} \sin ^{2} \alpha+\hat{\kappa}_{\perp} \cos ^{2} \alpha\right) \frac{\partial T}{\partial \theta}+\frac{\partial}{\partial r} \kappa_{\perp} \frac{\partial T}{\partial r}=n_{e} n_{C} L(T)
$$

where $\hat{\kappa}_{\perp}$ is the perpendicular transport within the flux tube. Transport perpendicular to the field occurs both across $\left(\kappa_{\perp}\right)$ and within the flux tubes $\left(\hat{\kappa}_{\perp}\right)$. Generally $\kappa_{\|} \sin ^{2} \alpha>>$ $\hat{\kappa}_{\perp} \cos ^{2} \alpha$ and we can ignore the $\hat{\kappa}_{\perp}$ term. In the vicinity of an x-point, however, $\sin \alpha \sim 0$ and the $\hat{\kappa}_{\perp}$ term cannot be ignored.

\section{III.A. Equilibrium}

Consider first radial equilibrium. If initially the equilibrium plasma is Marfe-free $(\partial T / \partial \theta \sim 0)$ there are two classes of radial equilibria: 1) The edge is sufficiently hot that low-Z impurities ( $\mathrm{C}, \mathrm{O}$, etc.) are fully stripped and do not radiate or b) low- $\mathrm{Z}$ impurity radiation dominates the edge power balance and prevents the edge temperature from rising above a level required to strip these impurities. Analysis of Eq. (1) without the parallel term in fact yield these two solutions $[8,11,12]$. In general a second equation for $p(x)$ must be solved consistently with Eq. (1a).

A Marfe may occur near the plasma edge in a layer of width $2 \delta$ bounded by flux tubes with mean radii respectively of $r_{a}$ and $r_{b}$, i.e. $r_{b}-r_{a} \sim 2 \delta$. The flux tubes at $r_{a}$ and $r_{b}$ are assumed to be at the respective temperatures $T_{a}$ and $T_{b}$. For $\delta<<r_{a}, r_{b}$ we can simplify Eq. (1) by differencing the cross-field conduction term as follows:

$$
\frac{\partial^{2} T}{\partial x^{2}} \rightarrow \frac{\left(T_{a}+T_{b}-2 T\right)}{\delta^{2}} \sim \frac{\left(T_{a}-2 T\right)}{\delta^{2}} .
$$

where for simplicity it is assumed that $T_{b}<<T_{a}$. We will first consider a single ordinary differential equation and afterwards consider two coupled ordinary differential equations. Eq. (4) retains the important effect that if the plasma within the layer of interest (in which a Marfe can occur) heats up, the cross-field power source into the layer decreases and visa versa. Furthermore, in a clean plasma without significant radiation cooling, the equilibrium is initially described by the non-radiating edge solution which requires $\kappa_{\perp} \partial^{2} T / \partial x^{2} \sim 0$ 
and Eq. (4) permits this result (when $T=T_{a} / 2$ ). We will therefore initially consider the following equation in our studies:

$$
\begin{gathered}
\left(\kappa_{\|} T_{s}\right)_{s}=-\kappa_{\perp} \frac{\left(T_{a}-2 T\right)}{\delta^{2}}+n_{e} n_{C} L(T) \\
T_{s}(0)=T_{s}(L)=0
\end{gathered}
$$

and $n_{e} T=p_{0}=$ constant. The subscripts indicate partial derivatives. We note that $\kappa_{\|}$ is generally a function of temperature (classically $\kappa_{\|}=\hat{\kappa_{\|}} T^{5 / 2}$ ). Following [10] we will approximate the radiation function by

$$
L(T)=L_{0} \frac{T^{3}}{T^{5}+T_{r a d}^{5}} .
$$

$T_{\text {rad }}$ represents the temperature at the peak of the radiation curve, i.e. for Carbon in local thermodynamic equilibrium $T_{\text {rad }}=8 \mathrm{eV}$. The details of this approximation do not effect the results and a more accurate form of the radiation function can be easily included. We will take $L_{0}=1.2 \times 10^{-31} \mathrm{Wm}^{3}, \hat{\kappa_{\|}}=1.29 \times 10^{22} \mathrm{~m}^{-1} \mathrm{~s}^{-1} \mathrm{eV}-5 / 2$ and $\kappa_{\perp}=3 n_{e} \mathrm{~m}^{-1} \mathrm{~s}^{-1}$.

\section{Solution by Method of Pseudo-Potential}

Equation (5) is a non-linear ordinary differential equation. It can be easily solved numerically and possesses multiple solutions. Valuable insight into the number and character of the solutions can be gained through the method of pseudo-potentials.

To apply this method we define the pseudo-potential by

$$
\phi(T)=-\int^{T}\left[n_{e} n_{C} L\left(T^{\prime}\right)-\frac{p_{0} \chi_{\perp}}{\delta^{2}}\left(T_{a} / T^{\prime}-2\right)\right] d T^{\prime}
$$

where $n_{e} \chi_{\perp} \equiv \kappa_{\perp}$. To include a temperature dependence, $\kappa_{\|} \propto T^{5 / 2}$ we could perform a change of variables $\hat{T}=T^{7 / 2}$ to eliminate the temperature dependence from the $\kappa_{\|}$term. 
This change of variables would distort the pseudo-potential without changing the relative positions of the maxima which determines the character of the solutions. For the present we ignore the temperature dependence of $\kappa_{\|}$and obtain from Eq. (5)

$$
\kappa_{\|} \frac{\partial^{2} T}{\partial s^{2}}=-\frac{\partial \phi}{\partial T}
$$

If we consider $\mathrm{s}$ as the pseudo-time co-ordinate and $\mathrm{T}$ as the pseudo-spacial co-ordinate Eq. (8) describes the motion of a ball of mass $\kappa_{\|}$on a potential hill described by $\phi(T)$. (The speed of the ball is $\partial T / \partial s \equiv T_{s}$ ). The ball will begin at some position $T_{i n i t}$ with zero speed and come back to a stop at time $=\mathrm{L}$ at position $T_{\text {final }}$. The extrema of the pseudo-potential, $\phi$, give rise to constant temperature (along-the-field-line) solutions. We will see that a result of the unusual boundary conditions is that the constant temperature equilibrium associated with the ball at a maxima is stable and equilibria at potential minima are unstable.

Fig. 1 displays a family of pseudo-potentials, drawn for a) low Carbon radiation, b) moderate radiation. The three extrema indicate (i) a constant (along-a field-line) solution at a warm edge temperature $\left(T=T_{3} \sim T_{a} / 2\right)$, (ii) a radiation burnout solution $T=$ $T_{1} \lesssim T_{\text {rad }}$ which corresponds to a detached plasma state, (iii) a solution having $T=$ $T_{2} \gtrsim T_{\text {rad }}$.

Consider the higher radiation pseudo potential curve displayed in Fig 1. In addition to the three constant temperature solutions just pointed out there will be a second class of solutions, the Marfe-like solutions such as shown in Fig. 2. For these solutions the pseudo-position varies with pseudo-time, or in other words $\mathrm{T}$ varies with s. An acceptable solution requires that a ball set at position $T=T_{i n i t}$, to the left of and close to $T_{3}$ will roll down the potential hill and stop in pseudo-time $\mathrm{t}=\mathrm{L}$ at $T=T_{\text {final }}$. This solution is shown in Fig. 2. Higher order solutions will also exist for a trajectory that will execute a finite number of passes through the position $T_{2}$ and have starting and ending positions $T_{\text {final }}<T<T_{\text {init }}$.

If the plasma is initially at the stable warm edge equilibrium solution $\mathrm{T}=T_{3}$ the system 
will make a transition to a new equilibrium when this solution is lost (referring to Fig 1 the warm edge temperature solution solution $T=T_{3}$ will evolve into the detached solution $T=T_{1}$ when the maxima at $T=T_{3}$ disappears due to increased radiation or reduced

edge temperature.) A more likely evolution path involves "jumping" between $T=T_{3}$ and a neighboring Marfe-like equilibrium brought about by edge temperature fluctuations [16-19]. The closest neighboring equilibrium will always be the lowest order Marfe.

Let us examine this possibility more closely. The maximum temperature point for the Marfe-like solution will approach $T_{3}$ as the field line length $\left(L \sim q_{a} R\right)$ increases. The two solutions will also approach each other as the warm temperature boundary condition $T=T_{a} \sim 2 T_{3}$ decreases, either due to an decrease of heating power into the core of the discharge or due to an increase of edge ion density.

In the vicinity of the x-point $L \rightarrow \infty$ and the high temperature end of the Marfe solution approaches the constant temperature solution, $T=T_{3}$ provided that $\phi\left(T_{1}\right) \geq$ $\phi\left(T_{3}\right)$. Therefore a transition can occur relatively easily at an $\mathrm{x}$-point when the field line length becomes long. (Near the x-point, however, the cross field transport within the flux tube will dominate the along-the-field-line transport and therefore the thermal transport within the flux tube will not completely disappear as the $\mathrm{x}$-point is approached.)

\section{III.B. Stability of Marfe Equations}

Returning to Eq. (8a) we now examine the stability of the Marfe-like solutions for $\mathrm{T}(\mathrm{s})$. If we perturb Eq. (8) $(T \rightarrow T+\delta T)$ we obtain for $\delta T$

$$
\gamma \delta T=\frac{\partial}{\partial s} \kappa_{\|} \frac{\partial \delta T}{\partial s}+\frac{d^{2} \phi}{d T^{2}} \delta T
$$

with $\gamma$ the growth rate. The boundary conditions on $\delta T$ are $\delta T_{s}(0)=\delta T_{s}(L)=0$ with $\delta T_{s} \equiv \partial \delta T / \partial s$. We can obtain a quadratic form by multiplying by $\delta T$ and integrating along the length of the field line: 


$$
\gamma \int_{0}^{L} \delta T^{2} d s=-\int_{0}^{L} \kappa_{\|} \delta T_{s}^{2} d s+\int_{0}^{L} \phi_{T T}(T) \delta T^{2} d s
$$

Eq. (10) can be shown to be maximizing and therefore and if a trial function is found that yields $\gamma>0$ this provides a sufficient condition for instability. Notice that the term containing $\kappa_{\|}$is always stabilizing whereas the term containing $\phi_{T T}$ can be either stabilizing or destabilizing. Recalling the form of the pseudo-potential (Fig. 1) the potential has negative $\phi_{T T}$ (stabilizing) regions in the vicinity of $T_{1}$ and $T_{3}$ and positive $\phi_{T T}$ in the vicinity of $T_{2}$.

Consider first the constant temperature solutions that occur at the extrema of the pseudo potential. Since $\phi_{T T}(T)$ is constant for these solutions it can be removed from the integral in Eq. (10). At the maxima $\phi_{T T}<0$ and therefore these solutions, $\left(T=T_{1}\right.$ or $T_{3}$ in Fig. 1) are stable. Consider next a potential minimum point ( $T=T_{2}$ in Fig. 1). If we take as a trial function $\delta T(s)=$ constant the stabilizing $\kappa_{\|}$term is eliminated and since $\phi_{T T}>0$ we obtain an instability. (Since Eq. (10) is a maximizing energy principle any trial function that leads to instability provides a sufficient condition for instability.) Therefore we find the somewhat counter intuitive result that constant temperature solutions at the maxima of the pseudo-potential are stable while those at the minima are unstable.

Consider next marfe-like equilibria. We again consider a trial function $\delta T(s)=$ constant to eliminate the stabilizing $\kappa_{\|}$term. We then obtain a sufficient condition for instability

$$
\int_{0}^{L} \phi_{T T} d s>0
$$

while a necessary condition for stability will be

$$
\int_{0}^{L} \phi_{T T} d s<0
$$

Referring to the potential in Fig. 1 we observe, for example, that a Marfe-like solution that sits entirely in the region where $\phi_{T T}>0$ would be unstable. The spacial dependence of 
$\phi_{T T}(T)$ however depends on the equilibrium solution for $T(s)$. Furthermore, since $-\phi_{T T}$ is the temperature derivative of the cross field and radiation function (RHS Eq. 5a) we see that stability requires that the field line average of the slope of the cross field and radiation function be positive.

From the pseudo-potential picture in which $\mathrm{T}$ corresponds to pseudo-position and $s$ to pseudo-time, a ball started off near a potential maxima $\left(\phi_{T T}>0\right)$ will spend a lot of time near the maxima. The edge temperature, $T_{a}\left(T_{3} \sim T_{a} / 2\right)$ must be sufficiently low for the ball to stop at time $t=L$. Thus a stable Marfe-like solution requires that the high temperature point be close to the maxima at $T=T_{3}$ so that a large region of the solution lies in the negative $\phi_{T T}$ region near $T_{3}$.

A solution with $T \lesssim T_{3}$ also requires that the pseudo-potential maxima satisfy the condition $\phi\left(T_{1}\right)>\phi\left(T_{3}\right)$. This imposes a necessary condition on the radiation term

$$
\int_{T_{1}}^{T_{3}} n_{e} n_{C} L(T) d T>\int_{0}^{L}\left(\kappa_{\perp} T_{x}\right)_{x} T_{s} d s \approx \int_{T_{1}}^{T_{3}} \frac{n_{e} \chi_{\perp}}{\delta^{2}}\left(T_{a}-2 T\right) d T
$$

Thus a stable Marfe-like equilibria with $T \lesssim T_{3}$ can occur as the impurity content, $n_{C}$ is increased or as the edge temperature, $T_{a}$ is reduced. The Marfe formation is also inhibited by an increase in $\chi_{\perp}$.

Further insight into the stability of the equilibria can be gained if we notice that $\phi_{T T}$ has a maximum in the vicinity of $T_{2}$. We can then obtain a second necessary condition for stability

$$
\int_{0}^{L} \kappa_{\|} \delta T_{s}^{2} d s>\phi_{T T}^{\max } \int_{0}^{L} \delta T^{2} d s
$$

If we consider, for example, a trial function that is localized in the destabilizing region (in which $\left.\phi_{T T}>0\right)$ of width $\Delta\left(\delta T_{s} \sim \delta T / \Delta\right)$ Eq. (13) approximately gives

$$
\kappa_{\|}>\phi_{T T}^{\max } \Delta^{2}
$$


Noting that $\Delta<L / 2$, we conclude that formation of a stable Marfe requires a sufficiently large parallel thermal conductivity, $\kappa_{\|}$.

Finally we note that an "inverse marfe" equilibrium can be a stable solution in the low radiation limit $\left(\phi\left(T_{1}\right)<\phi\left(T_{3}\right)\right)$. Referring to Fig. 1 ; for this solution we would start the ball off close to and to the right of $T=T_{1}$. The resulting solution for temperature would be close to $T_{1}$ along a large part of the field line length and only rise rapidly close at the other end of the field line (at the outside of the torus). Such equilibria would have a radiating region of much larger extent than an ordinary Marfe. Observation of these structures have not been reported in the literature.

If we consider that in the start up sequence of a tokamak, initially the plasma edge is warm it will be at the equilibrium point temperature $T=T_{3}$. If the edge is then cooled (due to the injection of gas or impurities) there will be a point when a stable Marfe-like solution with $T(L) \lesssim T_{3}$ will be produced and edge temperature fluctuations can cause the edge to "jump" into the Marfe equilibrium. Sufficient cooling can eliminate the pseudopotential maxima at $T=T_{3}$ and leave only the radiation collapse solution, $T=T_{1}$ which would be manifested as a detached plasma.

\section{III.C. Two Layer Model}

To consider the interaction between the flux layer near the $\mathrm{x}$-point and the adjoining scrape-off layer we consider two coupled equations for $T_{1}$ and $T_{2}$ which model these two respective flux layers:

$$
\begin{gathered}
\frac{\partial}{\partial s} \frac{\kappa_{\|}}{\zeta} \frac{\partial T_{1}}{\partial s}=-\kappa_{\perp} \frac{\left(T_{a}+T_{2}-2 T_{1}\right)}{\delta^{2}}+n_{e} n_{C} L\left(T_{1}\right) \\
\frac{\partial}{\partial s} \kappa_{\|} \frac{\partial T_{2}}{\partial s}=-\kappa_{\perp} \frac{\left(T_{1}+T_{\text {edge }}-2 T_{2}\right)}{\delta^{2}}+n_{e} n_{C} L\left(T_{2}\right)+P_{\text {recomb }}+n_{2} T_{2} \frac{d v_{\|}}{d s}
\end{gathered}
$$

with boundary conditions $\partial T_{1} / \partial s(s=0)=\partial T_{2} / \partial s(s=0)=\partial T_{1} / \partial s(s=L)=\partial T_{2} / \partial s(s=$ $L)=0$. 
In the $x$-point layer (Eq. 14a) $\zeta$ takes account of the relatively long field line length in the vicinity of the $x$-point (which effectively reduces parallel thermal conduction). The scrape off layer model equation (14b) contains a term for recombination and a flow term. These equations can be easily solved.

From the single layer problem discussed above we can assume that in each layer individually a radiation collapse is facilitated by a high impurity content, low temperature and low $\chi_{\perp}$. In the layer containing the $\mathrm{x}$-point it is also facilitated by a reduced thermal conductivity along-the-field-line. The two layers interact and a thermal collapse in one layer will reduce the cross field heat flow into the second layer. Thus the thermal collapse will tend to spread toward the edge. If the outer layers do not contain much pressure the observed radiation (which is proportional to $p^{2}$ ) will appear to decrease in the low pressure layer. A typical solution is shown in Fig. 3.

Recombination of the hydrogenic species becomes important when the temperature falls below $1 \mathrm{eV}$. It can be included as an additional term in the radiation function.

\section{Stability of 2-Dimensional Equilibria}

We can rewrite Eq. (1a) as

$$
\left(\kappa_{\|} T_{s}\right)_{s}+\left(\kappa_{\perp}(x) T_{x}\right)_{x}=f_{i m p} n_{e}^{2}(x) L(T)=p^{2} R(T)
$$

with $f_{i m p}$ the impurity fraction and $n_{e}(x)=p(x) / T$, i.e. pressure is constant along a field line and varies across flux surfaces. Eq. (15) will yield solutions similar to those discussed in the previous section. The stability of the solutions can be obtained from the perturbed form of Eq. (15)

$$
\gamma \delta T=\left(\kappa_{\|} \delta T_{s}\right)_{s}+\left(\kappa_{\perp}(x) \delta T_{x}\right)_{x}-p^{2}(x) R_{T} \delta T
$$


with boundary conditions $\delta T(a)=\delta T(b)=0$ at the radial boundaries of the flux layers and $\delta T_{s}(0)=\delta T_{s}(L)=0$ at either ends of the field lines. Integrating Eq (16) in $\mathbf{s}$ and $\boldsymbol{x}$ we obtain

$$
\iint \gamma \delta T^{2} d s d x=-\iint d s d x\left[\kappa_{\|} \delta T_{s}^{2}+\kappa_{\perp} \delta T_{x}^{2}+p^{2} R_{T} \delta T^{2}\right]
$$

As is well known [20-21] the negative slope region of the radiation function can drive instability while thermal conductivity is stabilizing. $R_{T}(T)$ is a function of space through the equilibrium relation. Eq. (17) illuminates a result of the simpler 1-D stability criterion (Eq. 11): if we choose a perturbation which zeroes the parallel transport, stability is determined by a competition between cross-field transport and radiation in the unstable region of the radiation function.

An interesting specia! case results when pressure is constant across the flux tubes, i.e. $p(x)=p_{0}$ and $\kappa_{\perp}$ is independent of $x$. (We are already assuming that pressure is constant along field lines). Taking this limit and assuming $T_{x}$ does not change sign $\left(T_{x}<0\right)$ we can take the $x$-derivative of Eq. (15) to obtain $R_{T}$ and substitute $R_{T}$ back into Eq. (17) to obtain

$$
\iint \gamma \delta T^{2} d s d x=-\iint d s d x\left[\kappa_{\|}\left(\delta T_{s}-\frac{T_{x s} \delta T}{T_{x}}\right)^{2}+\kappa_{\perp}\left(\delta T_{x}-\frac{T_{x x} \delta T}{T_{x}}\right)^{2}\right]
$$

Thus when pressure is constant and the radial temperature gradient does not change sign all equilibria are stable. In general the radial dependence of $\kappa_{\perp}\left(\kappa_{\perp} \approx n_{e}(x) \chi_{\perp}\right)$ and $p(x)$ adds additional terms to Eq. (18) and Eq. (17) must be considered to evaluate stability. The more stable nature of 2-dimensional equilibria indicates that an unstable 1-dimensional equilibrium may be stabilized when it is radially coupled to other more stable regions. 


\section{Conclusions}

By differencing the cross field conduction term we have reduced the thermal conductivity equation to one dimension (along-the-field-line) in a manner that still contains information about cross field transport. We observe that a stable Marfe-like equilibrium can form when the edge is cooled or when the edge impurity content increases sufficiently - An increase in $\chi_{\perp}$ will inhibit Marfe formation. Although both the constant temperature and the Marfe solutions are thermally stable, temperature fluctuations can cause a transition from one to the other. Furthermore a necessary condition for the stability of the Marfe (Eq. 11b) requires that the temperature remain close to the warm constant temperature equilibrium solution throughout most of the field line length and then fall quickly into the strongly radiating temperature range. This condition eliminates a gradual temperature drop solutior: which may be more desirable for spreading the radiation energy in some divertor scenarios.

Two types of Marfe solutions are observed: In the low impurity concentration case $\phi\left(T_{1}\right)>\phi\left(T_{3}\right)$ and the Marfe forms in the cool end of the temperature range with $T_{1} \lesssim T<<$ $T_{3}$. In the high impurity concentration case $\phi\left(T_{1}\right)<\phi\left(T_{3}\right)$ the Marfe forms at the warm end of the temperature range with $T_{1}<<T \lesssim T_{3}$. The latter case represents the Marfe solution that can most easily transition from the warm constant temperature solution.

We have also approximated the 2-D thermal diffusion equation with coupled 1-D equations. An examination of the stability of the 2-D fluid equations indicates that perpendicular coupling can enhance the stability. In the special case of constant pressure and when the cross flux temperature gradient does not change sign we have seen that all equilibria are stable.

The approach taken in this study illustrates the qualitative features of edge/scrape-off layer phenomena. More detailed calculations can be obtained from two dimensional codes [22-24]. We observe that because multiple solutions exist simulation codes can miss the correct solution. Additionally although time dependent codes can follow the evolution of unstable to stable equilibria they cannot predict jumps between neighboring stable 
solutions.

Although in principle Marfes would be avoided in sufficiently pure hydrogenic plasmas only small fractions of low $\mathrm{Z}$ impurities are required for their formation. It therefore appears unlikely that a strong cooling of the plasma edge (i.e. to form a radiative divertor) can occur without the formation of a Marfe. In principle a tokamak reactor could take advantage of this tendency by using an $\mathrm{x}$-point Marfe to radiate a significant fraction of the power leaving the plasma. In a double null (symmetric up/down) divertor it may be possible to create two Marfes (above and below the plasma) which would increase the power radiated and distribute it more evenly on the reactor first wall.

\section{Acknowledgements}

The authors would like to thank S. Krasheninnikov for valuable suggestions. This work was performed under contract to the USDOE. 


\section{References:}

1 J. Terry et al., Bull APS 26, 886 (1981).

2 D.R. Baker et al.,Nucl. Fus. 22, 807 (1982).

3 F. Alladro et al., Phys. Lett. A, 90, 405 (1982).

4 B. Lipschultz et al., Nucl. Fus. 24, 977 (1984).

5 B. Lipschultz, J. Nucl. Mater, 145, 15 (1987).

6 J. Terry et al., Bull APS 38, 1955 (1993).

7 T.W. Petrie et al., Bull APS 38, 2071 (1993).

8 J. Neuhauser et al., Nucl. Fus. 26, 1679 (1986).

9 T.E. Stringer, Proc 12th EPS Conf on Cont Fus and Plasma Physics, Budapest 1, 86 (1985).

10 D. McCarthy and J.F. Drake, Phys Fluids B 3, 22 (1991).

11 S.I. Krasheninnikov, JETP Lett., 5, 320 (1988).

12 J.A. Wesson and T.C. Hender, "Theory of Marfe Stability", JET report JET-P(92)12 (1992), submitted to Nucl. Fus.

13 J.F. Drake, Phys Fluids 30, 2429 (1987).

14 B.Meerson, N.Petviashvili, T.Tajima, "Marfes in Tokamak Edge Plasma: Pattern Formation Under Non-local Constraints", Report IFSR 658, Institute for Fusion Studies, University of Texas, Austin (1994), submitted to Phys. Fluids (1994).

15 S.I. Braginskii, in Reviews of Plasma Physics, edited by M.A. Leontovich (Consultants Bureau, N.Y., 1965) Vol. I, p.205.

16 R. Watterson, R.E. Slusher, C.M. Circo, Phys. Fluids 28, 2857 (1985). 
17 T. Crowley and E. Mazzucato, Nucl. Fusion 25, 507 (1985).

18 S. Zweben and R.J. Taylor, Nucl. Fusion 23, 513 (1983).

19 C.P. Ritz et al., Phys. Fluids 27, 2956 (1984).

20 E.N. Parker, Astrophys. J., 117, 431 (1953).

21 G.B. Field, Astrophys. J., 142, 531 (1965).

22 B. Braams et al., J. Nucl. Mater., 121, (1984) 75.

23 D. Knoll et al., J. Comp. Physics, (1992).

24 T. Rognlien et al., J. Nucl. Mater., 196-198, (1992) 346. 


\section{Figure Captions:}

FIG. 1. Typical pseudo-potential curves, $\phi$ vs. T for a) low impurity (Carbon) content and b) moderate impurity content.

FIG. 2. Marfe-like equilibrium that results from the high radiation pseudo potential shown in Fig 1.

FIG. 3. Two layer solution showing a Marfe that extends in both the x-point and scrapeoff layers. 


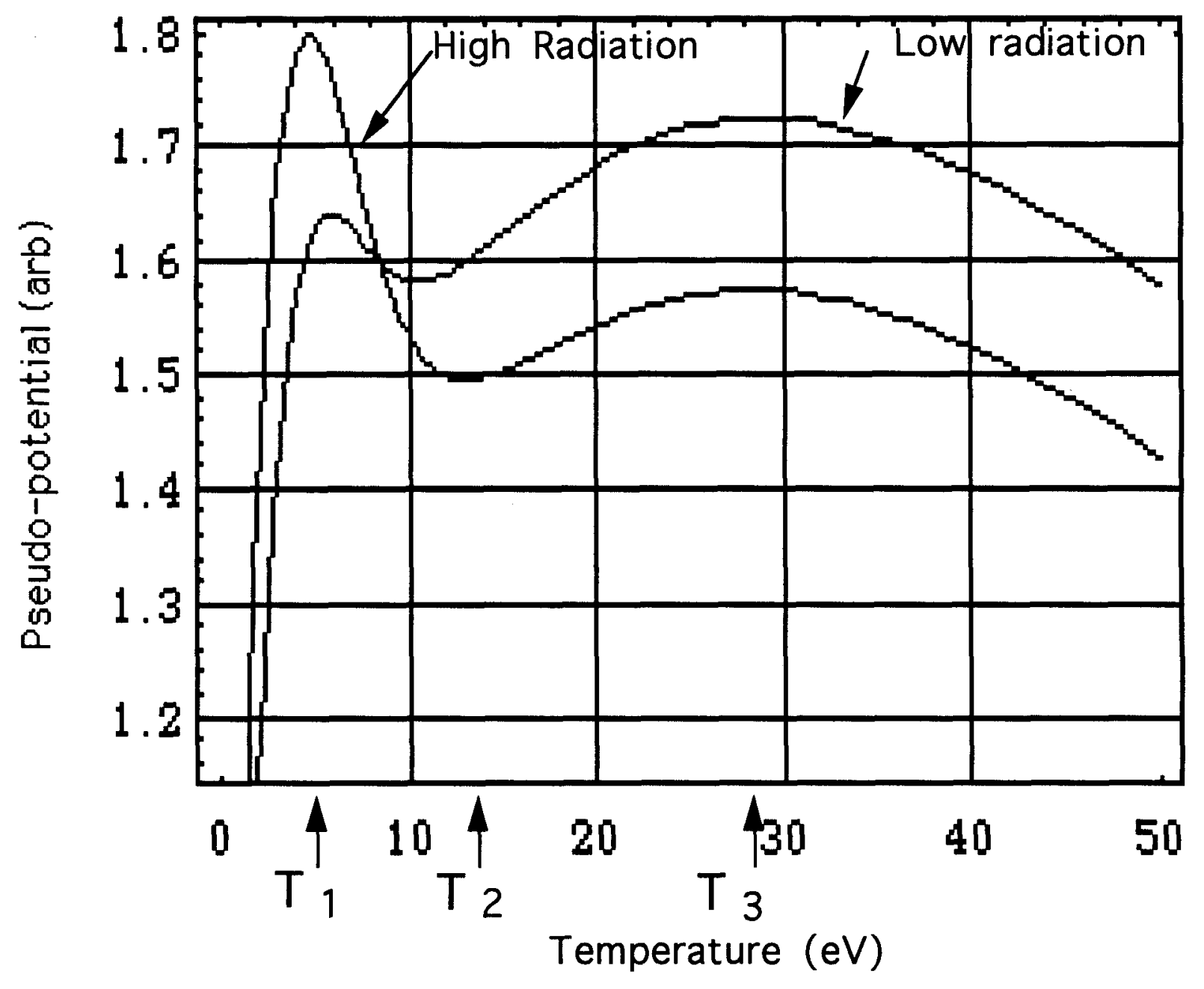

Fig 1 


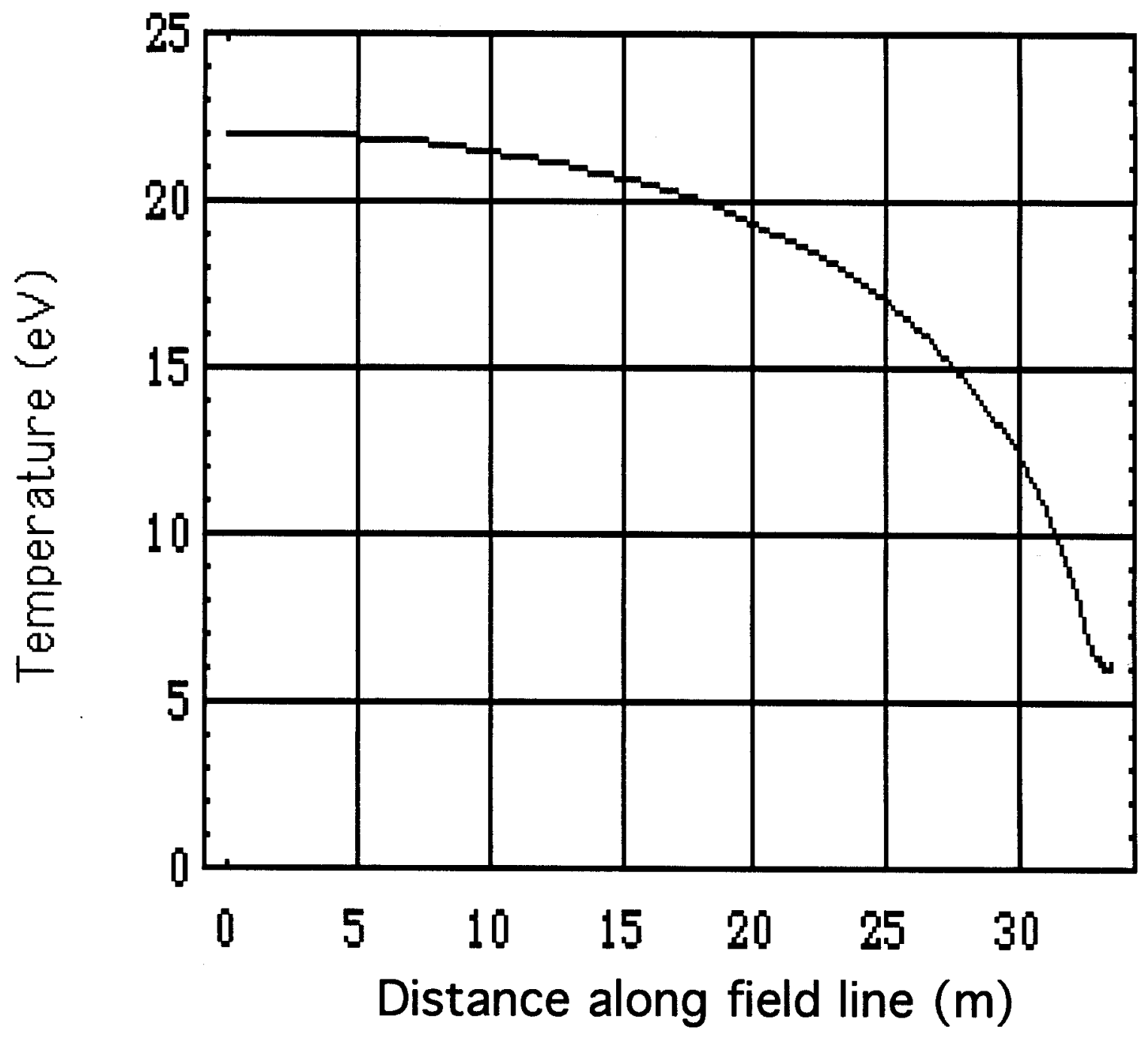

Fig 2 


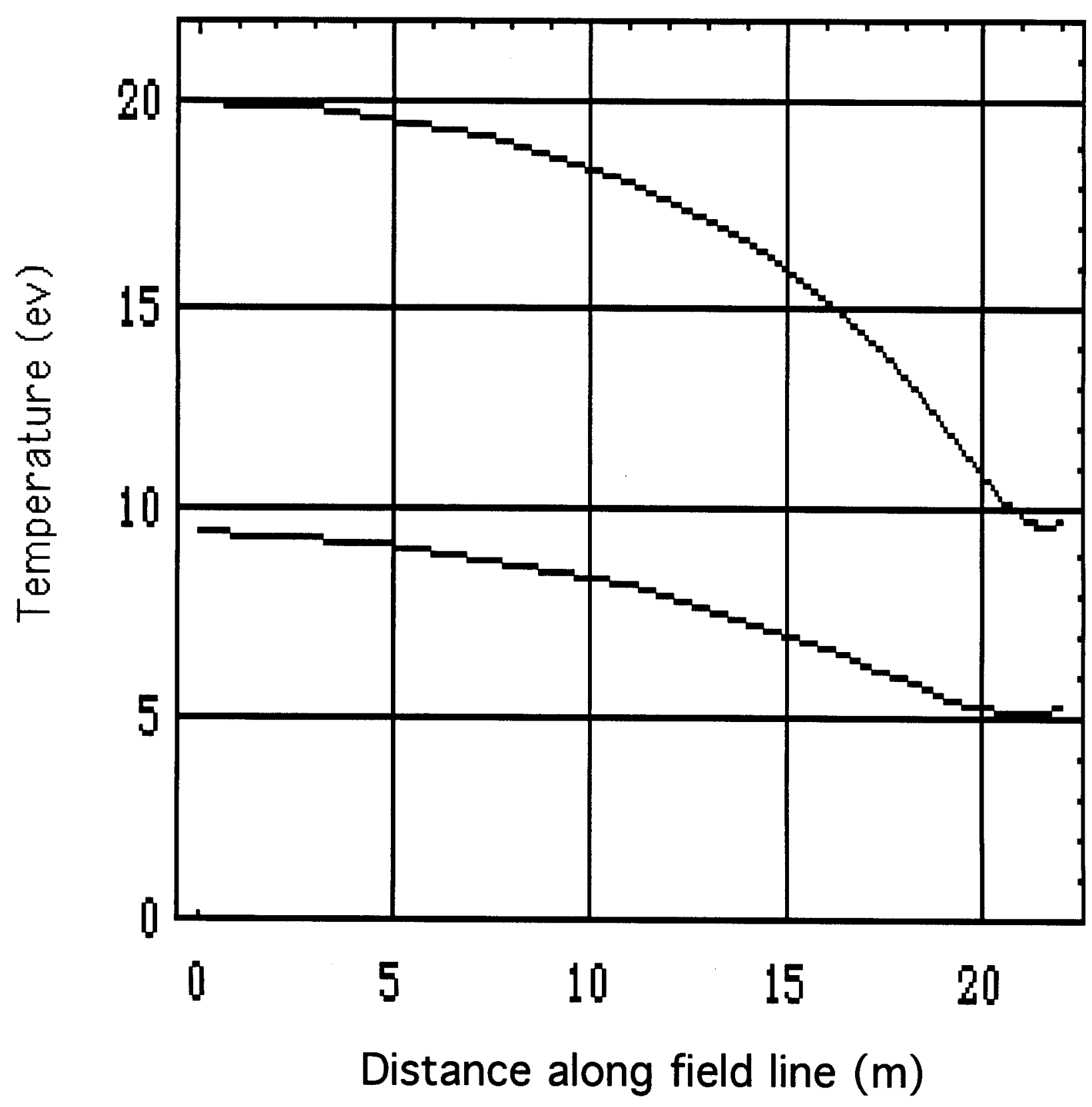

Fig 3 